\title{
Altered Cell Viability and Proliferation Activity of Peripheral Lymphocytes in Patients with Alzheimer's Disease
}

\author{
Se Chang Yoon ${ }^{1}$ \\ Young-Ah Kwon ${ }^{2}$ \\ Hyeran Kim ${ }^{1}$ \\ Seonwoo $\mathrm{Kim}^{2}$ \\ Sangmee Ahn $\mathrm{Jo}^{3}$ \\ Doh Kwan Kim ${ }^{1,2} \bowtie$ \\ 'Department of Psychiatry, \\ Samsung Medical Center, \\ Sungkyunkwan University \\ School of Medicine, Seoul, \\ ${ }^{2}$ Center for Clinical Research and \\ Biostatistics Unit, \\ Samsung Biomedical \\ Research Institute, Seoul, \\ ${ }^{3}$ Department of Biomedical Science, \\ National Institute of Health, \\ Seoul, Korea
}

Objective We evaluated cell viability and proliferation activity of peripheral lymphocytes as potential models of neuronal death in Alzheimer's disease (AD).

Methods We analyzed the cell viability and proliferation activity of phytohemagglutinin (PHA)-activated lymphocytes from $68 \mathrm{AD}$ patients and 33 normal controls. The cellular measures were made at baseline ( 0 hr), 24 hrs, 48 hrs, $72 \mathrm{hrs,}$, and $96 \mathrm{hrs}$ after PHA stimulation.

Results Cell viability in the AD patients was significantly decreased at 72 hrs and 96 hrs, compared with the normal controls. The declining ramp of the proliferation activity from 48 hrs to 72 hrs after PHA stimulation was significantly related to cell viability at 72 hrs and at $96 \mathrm{hrs}$ in the AD patients.

Conclusion Lymphocytes from patients with $\mathrm{AD}$ have altered viability and proliferation characteristics in culture following PHA stimulation. These findings suggest that lymphocytes may be used as a peripheral tissue model of cell cycle dysregulation in AD.

Psychiatry Investig 2010;7:68-71

Key Words Alzheimer's disease, cell cycle, Biomarker, Cell death, Peripheral lymphocytes.

Received: October 27, 2009 Revised: February 4, 2010 Accepted: February 17, 2010

Available online: March 3, 2010

\section{Introduction}

Alzheimer's disease (AD), which is the most frequent cause of dementia among elderly people, is a progressive neurodegenerative disorder manifested by cognitive and memory deterioration, a variety of neuropsychiatric symptoms and behavioral disturbances, and progressive impairment of activities of daily living. There are currently no curative treatments that can reverse the disease progression of $\mathrm{AD}$, so early diagnosis and slowing down the disease progression with some effective drugs is the best way to treat AD patients. ${ }^{1}$ Neurofibrillary tangles and neuritic amyloid plaques represent the core neuropathologic features of AD. The precise factors responsible for the development of these lesions remain enigmatic, but the invariant consequence is the death of neurons. Neuronal death is the result of the process termed apoptosis, a complex cascade of intracellular events. ${ }^{2,3}$ It has been suggested that peripheral cells, such as fibroblasts or cells in body fluids, can provide biological markers for research into the pathophysiology of AD. ${ }^{4}$

During the process of immortalizing peripheral lymphocytes in our genome research into $\mathrm{AD}$, we incidentally observed that lymphocytes from patients with $\mathrm{AD}$ showed poor proliferative activity, compared with normal controls. The lymphocytes of AD patients showed shrinking and reduced viability, which decreased rapidly 3-4 weeks after their immortalization by the Epstein Barr virus. Previous study results from other investigators already demonstrated that the neuronal apoptotic mechanism may be involved in pathophysiology of AD. ${ }^{2,3} \mathrm{Ba}-$ sed on our fortuitous observations, we have examined the hypothesis that the apoptotic pathophysiology in $\mathrm{AD}$ patients is reflected in peripheral nucleated cells (lymphocytes) in the form of alterations in the cell viability and proliferation activity. We compared the cell viability and 
proliferation activity of peripheral lymphocytes following mitogenic stimulation in $\mathrm{AD}$ patients and normal controls.

\section{Methods}

\section{Subjects}

Patients were recruited from the Clinical Trials Programs of the Samsung Medical Center Geropsychiatry Clinic. Details of the diagnostic procedure, and inclusion and exclusion criteria are provided in a previous publication. ${ }^{5}$ All patients received a comprehensive neuropsychological screening battery, which cannot be detailed in this report. Severity of dementia was assessed with the Korean version of the MiniMental State Examination (K-MMSE), ${ }^{6}$ Seoul-Instrumental Activity of Daily Living (S-IADL), 7 and the Clinical Dementia Rating (CDR) scale. ${ }^{8}$ In our Geropsychiatry Clinic, the inter-observer diagnostic reliability of $\mathrm{AD}$ and non-AD dementias is $90.3 \%$. Brain magnetic resonance imaging and electroencephalography were performed in all cases to exclude other possible causes of dementia. Patients with other neurologic or psychiatric disorders or clinically significant medical conditions were excluded. No patient had a history of head trauma, or alcohol or drug abuse. Healthy volunteers with no history of memory impairment or psychiatric illness were recruited as normal controls by advertisement, and were matched to the patient group by age, sex and educational status. They were screened by the K-MMSE (K-MMSE score 27 or higher) and Minnesota Multiphasic Personality Inventory (on which they had validity and clinical scale scores ranging from 35 to 65 ). The protocol was approved by the ethics review board of Samsung Medical Center, Seoul, Korea. Signed informed consent was obtained from all participants. Data from five subjects (four from $\mathrm{AD}$ patients and one from normal control) were excluded from analysis because of insufficient amount of specimen. In the final sample, $68 \mathrm{AD}$ patients and 33 normal controls were included for data analyses.

\section{Measurement of the viability and proliferation activity of lymphocytes}

Venous blood was drawn between $0800 \mathrm{~h}$ and $1000 \mathrm{~h}$ for the separation of peripheral lymphocytes. The blood samples were heparinized, stored at $4^{\circ} \mathrm{C}$ immediately after venipuncture, and assayed within $24 \mathrm{hrs}$. Blood mononuclear cells were isolated by centrifugation at $300 \mathrm{~g}$ for $15 \mathrm{~min}$ using the FicollHypaque (Pharmacia Biotech, Uppsala, Sweden) density gradient technique. The cells were washed two times with phosphate buffered saline (PBS), and then the lymphocytes were separated with Hank's Balanced Salt Solution. The lymphocytes obtained from patients and controls were cultured spontaneously in RPMI1640 (GIBCO) containing 10\% fetal bovine serum (FBS) at $37^{\circ} \mathrm{C}$ with $5 \% \mathrm{CO}_{2}$ after adding $20 \mu \mathrm{g} /$ $\mathrm{mL}$ of phytohemagglutinin (PHA, GIBCO), then measured as indicated below for the assessment of the viability and proliferation activity at various time points, viz. baseline (0 hrs), $24 \mathrm{hrs}, 48 \mathrm{hrs}, 72 \mathrm{hrs}$ and $96 \mathrm{hrs}$.

Quantitative determination of cell viability was performed using the live/dead assay by the trypan blue method. The $0.4 \%$ trypan blue solution (SIGMA) was added to the PHA-activated cells at different time points and then incubated at room temperature for $10 \mathrm{~min}$. The blue-stained cells and non-stained cells were counted twice by means of a hemocytometer (live cells, not stained). Cell viability was calculated as percentage of viable cells to total cell number. The proliferation activity of the cells was determined by measuring the BrdU proliferation activity (Cell Proliferation ELISA, BrdU colorimetric kit, Roche, Mannheim, Germany). The PHA-activated cells at different time points were incubated at $37^{\circ} \mathrm{C}$ for $2 \mathrm{hrs}$, after adding $10 \mu \mathrm{L} /$ well of BrdU labeling solution. The supernatants were separated by centrifugation at $300 \mathrm{~g}$ for $10 \mathrm{~min}$. The pellets were dried at $60^{\circ} \mathrm{C}$ for $1 \mathrm{hr}$, incubated at room temperature for $30 \mathrm{~min}$ after adding FixDenat solution $200 \mu \mathrm{L} /$ well, and then washed. $100 \mu \mathrm{L} /$ well of Anti-BrdU POD working solution was added, and the wells were incubated at room temperature for $90 \mathrm{~min}$, then washed three times to separate the conjugate antibody. $100 \mu \mathrm{L} /$ well of TMB substrate was added for the fluorescent reaction, and the wells incubated at room temperature for $30 \mathrm{~min}$. The reaction was stopped by adding $25 \mu \mathrm{L}$ of $1 \mathrm{M} \mathrm{H}_{2} \mathrm{SO}_{4}$. The fluorescence intensity was measured by means of an ELISA reader at 450 $\mathrm{nm}$ (reference wavelength $690 \mathrm{~nm}$ ).

\section{Statistical analysis}

The independent two-sample t-test and Mann-Whitney test were used to evaluate the difference in the cell viability/proliferation activity of the peripheral lymphocytes of the patients and controls at each time point. Non-parametric methods were applied to the statistical analyses of the variables, which were not normally distributed. The correlation between the cell viability and proliferation activity of the lymphocytes in AD patient group was examined using Spearman's correlation analysis with Bonferroni's correction. Relationships between the cell viability/proliferation activity of the lymphocytes and KMMSE, S-IADL and CDR scores in AD patient group were tested using Spearman's correlation analysis with Bonferroni's correction.

\section{Results}

Subject groups were of similar age (AD 72.1 \pm 9.5 years, control 71.3 \pm 5.2 years, $\mathrm{p}=0.29$ ), female/male ratio ( $A D$ 49/19, control 22/11, $\mathrm{p}=0.58)$ and educational status (AD 8.4 \pm 5.1 years, control $8.3 \pm 4.4, \mathrm{p}=0.93$ ). The $\mathrm{AD}$ group had mild to severe dementia with a mean K-MMSE score of 14.6 \pm 7.3 (range 1,26 ) and a mean CDR score of $1.51 \pm 0.85$ (range 1,3 ) by 
CDR. The microscopic comparison of the viability of the peripheral lymphocytes from the patient with $\mathrm{AD}$ and normal controls, cultured for 96 hours in RPMI1640 after adding PHA, is illustrated in Figure 1. The lymphocytes from the patients with $\mathrm{AD}$ had different viability characteristics than control lymphocytes after stimulation with PHA.

During the early phase of lymphocyte culture, we did not observe any difference of cell viability between the two groups. The cell viability at $24 \mathrm{hrs}(91.5 \pm 5.8 \%$ vs. $91.2 \pm 5.4 \%$, $\mathrm{p}=1.0000)$ and $48 \mathrm{hrs}(89.8 \pm 6.1 \%$ vs. $91.4 \pm 4.4 \%, \mathrm{p}=1.0000)$ after PHA stimulation showed no difference between the AD and control group. However, at later time points, the cell viability of the lymphocytes from the AD group decreased more rapidly and showed a significant reduction at $72 \mathrm{hrs}(78.9 \pm 8.9 \%$ vs. $85.4 \pm 7.8 \%, \mathrm{p}=0.002)$ and $96 \mathrm{hrs}(70.7 \pm 12.1 \%$ vs. $83.3 \pm$ $9.8 \%, \mathrm{p}=0.0004)$ after PHA stimulation, compared to the control group (Figure 2A). The proliferation activity detected in the AD group did not differ significantly from that in the control group at any time point. In $\mathrm{AD}$ group, the proliferation activity tended to be higher than that in the control group at 24 hrs (median, interquartile interval ; $0.37,0.17-0.56 \%$ vs. 0.23 , $0.13-0.35 \%, \mathrm{p}=0.0796)$ and $48 \mathrm{hrs}(0.50,0.35-0.73 \%$ vs. 0.42 , $0.30-0.62 \%, p=0.1668)$, then showed a rapid decline by $96 \mathrm{hrs}$ (declining ramp of proliferation from $72 \mathrm{hrs}$ to $96 \mathrm{hrs} ;-0.08$, $-0.15--0.03 \% / \mathrm{hr}$ vs. $-0.01,-0.12-0.02 \% / \mathrm{hr}, \mathrm{p}=0.011$ )(Figure 2B). The decline of the proliferation activity appeared to be earlier in the $\mathrm{AD}$ than that in the control group. The declining ramp of the proliferation activity from $48 \mathrm{hrs}$ to $72 \mathrm{hrs}$ after PHA stimulation was significantly correlated with cell viabil- ity at $72 \mathrm{hrs}(\rho=0.442, \mathrm{p}<0.001)$ and at $96 \mathrm{hrs}(\rho=0.436$, $\mathrm{p}<0.001)$ in AD patients. There was no correlation between the cell viability/proliferation activity of lymphocytes and clinical scores reflecting severity of dementia (K-MMSE, S$\mathrm{IADL}, \mathrm{CDR}$ ) in the $\mathrm{AD}$ group (data not shown).

\section{Discussion}

We demonstrated that the peripheral lymphocytes from patients with $\mathrm{AD}$ had altered viability during culture stimulated with PHA, compared to those from controls. The cell viability of lymphocytes in $\mathrm{AD}$ patients decreased more rapidly after mitogenic stimulation than in normal controls (Figures 1 and 2).

The specific mechanisms responsible for this poor viability and premature death of lymphocytes in $\mathrm{AD}$ patients remain to be elucidated. Previous studies reported various biological disturbances in lymphocytes of AD patients, including cell cycle dysregulation and alterations of the immune system. ${ }^{9-11} \mathrm{~A}$ strong possibility is that the cell cycle dysregulation may be linked with this premature death of peripheral lymphocytes in $\mathrm{AD}$. The relation between abnormal cell cycle regulation, neuronal cell death and the pathogenesis of neurodegenerative diseases such as AD has been well documented. ${ }^{12}$ Peripheral lymphocytes, as in the case of neurons in the adult brain, are paused in the quiescent phase of the cell cycle, in which they are differentiated but not proliferating. The reactivation of the cell cycle by mitogenic stimuli (PHA in our study) leads to cell proliferation. Nagy et al. ${ }^{13}$ reported abnormal cell cycle kine-
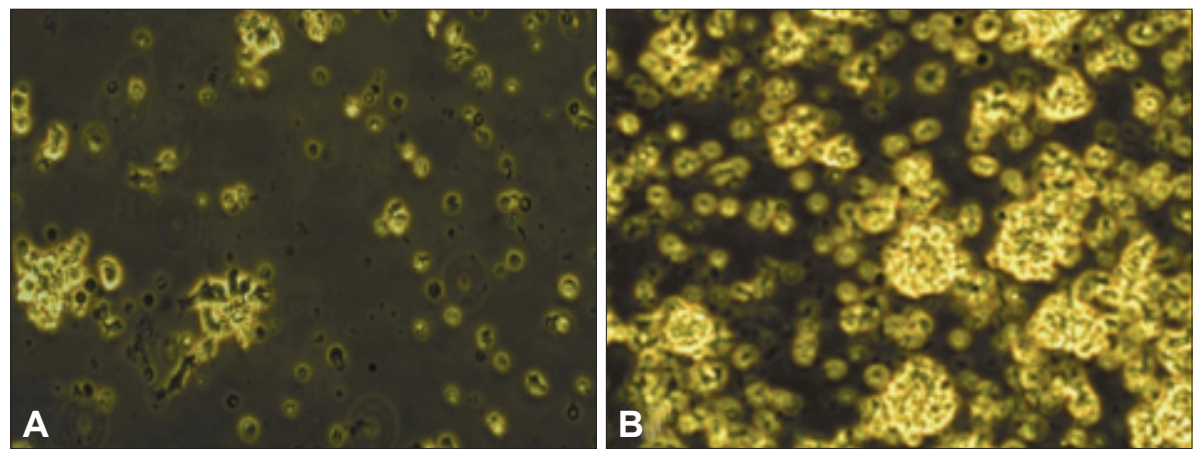

Figure 1. Comparison of the viability of peripheral lymphocytes from (A) patients with Alzheimer's disease and (B) normal controls, cultured for 96 hours in RPMI1640 after adding phytohemagglutinin. The bright yellow fluorescence indicates live lymphocytes.
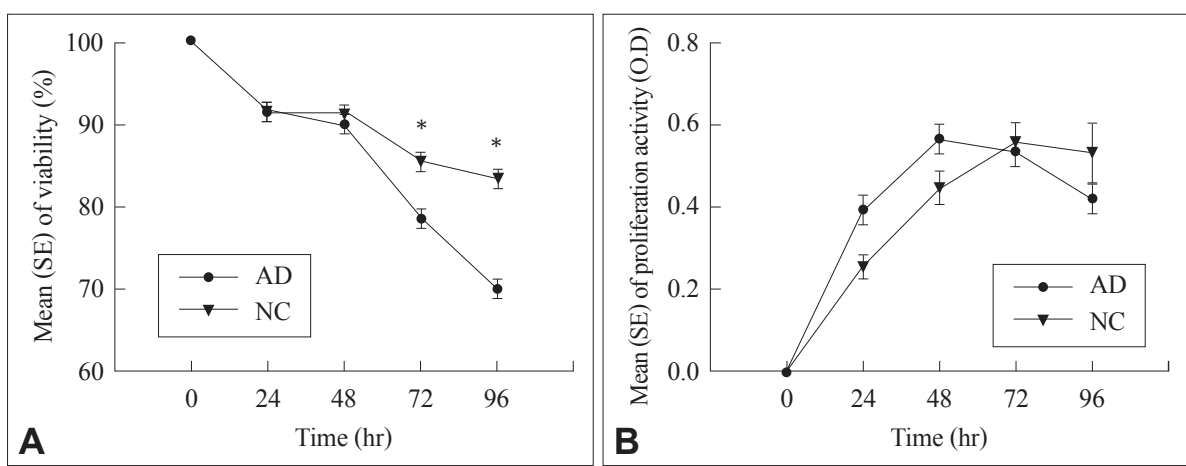

Figure 2. A: Cell viability of phytohemagglutinin-activated lymphocytes from patients with $A D$ and NC. B: Proliferation activity of phytohemagglutinin-activated lymphocytes from patients with $A D$ and NC. ${ }^{*} p<0.05$. SE: standard error, O.D: optical densities, AD: Alzheimer's disease, NC: normal controls. 
sis in the lymphocytes of $\mathrm{AD}$ patients based on the in vitro responsiveness to a G1 inhibitor (rapamycin). We observed that the declining ramp after gradual increase of the proliferation activity was significantly correlated with cell viability in the later phase. Our finding also supports the hypothesis that the cell cycle dysregulation in peripheral lymphocytes precedes subsequent cell death. In addition, an alteration of the immune system itself may contribute to the poor viability of lymphocytes in $\mathrm{AD}$ patients. A difference in the T cell subpopulations, ${ }^{9}$ a role by the immunity receptor such as $\mathrm{CD} 14^{10}$ and the decreased expression of an early proliferation marker of lymphocytes ${ }^{11}$ have been reported in $\mathrm{AD}$ patients.

We did not find any relationship between the cell viability/ proliferation activity of lymphocytes and clinical scores reflecting disease severity in the AD group. However, this result is in agreement with those of previous studies regarding cell cycle dysregulation as a biomarker of an early event in AD. ${ }^{11,12}$ In view of this lack of correlation with disease severity, it is possible that the alterations of lymphocyte viability or proliferation activity in $\mathrm{AD}$ patients may appear early, before the onset of clinical dementia symptoms.

The cell viability and proliferation activity in the peripheral lymphocytes of AD patients were altered in patients with established probable AD. These preliminary finding suggests that lymphocytes may be used as a peripheral tissue model to develop biomarker of AD.

\section{- Acknowledgments}

This study was supported by a grants from Choi Shin-Hai Neuropsychiatric Research Fund, the Korea Health 21 R\&D Project, Ministry of Health \& Welfare (A040042 and A060618), and National Research Foundation of Korea funded by the Korea government (MEST, R0A 2007-000-20129-0).

\section{REFERENCES}

1. Cummings JL, Cole G. Alzheimer disease. JAMA 2002;287:23352338.

2. Cotman CW, Anderson AJ. A potential role for apoptosis in neurodegeneration and Alzheimer's disease. Mol Neurobiol 1995;10:19-45.

3. Rissman RA, Poon WW, Blurton-Jones M, Oddo S, Torp R, Vitek MP, et al. Caspase-cleavage of tau is an early event in Alzheimer disease tangle pathology. J Clin Invest 2004;114:121-130.

4. Gasparini L, Racchi M, Binetti G, Trabucchi M, Solerte SB, Alkon D, et al. Peripheral markers in testing pathophysiological hypotheses and diagnosing Alzheimer's disease. Faseb J 1998;12:17-34.

5. Kim DK, Seo MY, Lim SW, Kim S, Kim JW, Carroll BJ, et al. Serum melanotransferrin, p97 as a biochemical marker of Alzheimer's disease. Neuropsychopharmacology 2001;25:84-90.

6. Folstein MF, Folstein SE, McHugh PR. "Mini-mental state". A practical method for grading the cognitive state of patients for the clinician. J Psychiat Res 1975;12:189-198.

7. Ku HM, Kim JH, Kwon EJ, Kim SH, Lee HS, Ko HJ, et al. A study on the reliability and validity of Seoul-Instrumental Activities of Daily Living (S-IADL). J Korean Neuropsychiatr Assoc 2004;43:189-199.

8. Morris JC. The Clinical Dementia Rating (CDR): current version and scoring rules. Neurology 1993;43:2412-2414.

9. Robinson Agramonte M, Dorta-Contreras AJ, Lorigados Pedre L. Immune events in central nervous system of early and late onset Alzheimer's disease patients. Rev Neurol 2001;32:901-904.

10. Fassbender K, Walter S, Kühl S, Landmann R, Ishii K, Bertsch T, et al. The LPS receptor (CD14) links innate immunity with Alzheimer's disease. Faseb J 2004;18:203-205.

11. Stieler JT, Lederer C, Brückner MK, Wolf H, Holzer M, Gertz HJ, et al. Impairment of mitogenic activation of peripheral blood lymphocytes in Alzheimer's disease. Neuroreport 2001;12:3969-3972.

12. Becker EB, Bonni A. Cell cycle regulation of neuronal apoptosis in development and disease. Prog Neurobiol 2004;72:1-25.

13. Nagy Z, Combrinck M, Budge M, McShane R. Cell cycle kinesis in lymphocytes in the diagnosis of Alzheimer's disease. Neurosci Lett 2002;317:81-84. 